\section{PERFORMANCE ANALYSIS OF PURE PARAFFIN WAX AS RFID TAG SUBSTRATE}

\author{
S. Manzari, ${ }^{1}$ A. A. Babar, ${ }^{1}$ L. Ukkonen, ${ }^{1}$ Atef Z. Elsherbeni, ${ }^{2}$ \\ G. Marrocco, ${ }^{3}$ and L. Sydänheimo ${ }^{1}$ \\ ${ }^{1}$ Rauma Research Unit, Department of Electronics, Tampere \\ University of Technology, Rauma 26100, Finland; Corresponding \\ author: sabinamanzari@virgilio.it \\ ${ }^{2}$ Department of Electrical Engineering, The University of \\ Mississippi, MS 38677-1848 \\ ${ }^{3}$ Dipartimento di Informatica, Sistemi e Produzione, Università di \\ Roma Tor Vergata, Roma 00133, Italy
}

Received 5 May 2011

ABSTRACT: The article presents an investigation of the performance of the Paraplast, a kind of pure paraffin wax, used as a substrate of RFID tag antennas operating at UHF RFID band. To achieve this purpose, two similar RFID tag antennas with same dimensions have been simulated, fabricated and tested. These include a simple dipole tag on a Rogers RT/Duroid 5880 substrate and on pure paraffin substrate. Because of the relatively close dielectric constant values, paraffin substrate is compared with Duroid RT5880. Paraffin wax can be considered as a low cost and low loss substrate. The main purpose in the future will be using paraffin as a passive temperature sensor, by exploiting the changes in molecular structure, volume and dielectric properties with temperature, especially near its melting point. (C) 2011 Wiley Periodicals, Inc. Microwave Opt Technol Lett 54:442-446, 2012; View this article online at wileyonlinelibrary.com. DOI 10.1002/ mop. 26592

Key words: paraffin wax; passive temperature sensor; RFID

\section{INTRODUCTION}

A passive RFID system is composed of two main components: a digital device called tag, that includes an antenna and an IC-chip, which works like a label and the radio scanner device called reader. The tag carries the identification data saved inside its memory, whereas the reader interrogates the passive tag by means of radio-frequency electromagnetic signals. In this way, the unique identification code (ID) can be wirelessly transferred between the two devices [1].

The RFID technology is nowadays used in logistics, inventory management, manufacturing, and security. Some initial applications are currently experimented in sensoristics and bioengineering [2-6].

In addition to these common usages of RFID, an analog processing of the physical signals related to the reader-tag communication could permit to achieve much more information about the target without the need of additional electronics. Passive sensors can be integrated into the antenna design for this purpose. Also, it is possible to analyze the changes of the tag's behavior and performance with the physical and geometrical features of a real target. It is well experienced that when the same tag is placed on different targets or in the presence of different physical conditions, the tag antenna's input impedance will be subjected to a change. Consequently, a change in the reading distance and in the backscattered power can be observed.

Tag performance is greatly affected by the losses in the substrate material. The lower the substrate losses, the higher the performances of the tag antenna in terms of gain and reading range can be.

In this article, the behavior of two-folded dipoles tags operating at UHF RFID band is compared and analyzed. The first one is constructed on a layer of Duroid RT5880 and the second one on a layer of pure paraffin wax.

This work represents the first step to consider the paraffin as an antenna's substrate. Because of the changes in the dielectric properties of paraffin as a function of temperature, it can be used in future passive temperature sensor applications.

In Section 2, the main characteristics of the paraffin wax are introduced. Section 3 describes the proposed tag antenna and the first simulations results. In Section 4, the experimental results and the comparison between the two tags are discussed.

\section{PARAFFIN WAX CHARACTERISTICS}

The term paraffin wax is used to define mixtures of various hydrocarbon groups, especially paraffins and cycloalkanes, that are solid at the ambient temperature and have low viscosity. The general formula that defines paraffins is $\mathrm{C}_{n} \mathrm{H}_{2 n}+2$ with $n$ between 20 and 40 range [7].

Paraffin wax is mostly found as a white odorless, tasteless, waxy solid, with a typical melting point between 40 and $70^{\circ} \mathrm{C}$ and a density of around $0.9 \mathrm{~g} / \mathrm{cm}^{3}$. Pure paraffin wax is an excellent insulator, with high electrical resistivity between $10^{16}$ and $10^{18} \Omega / \mathrm{cm}^{3}$.

Paraffin tends to expand when it melts, decreasing its density. According to Ref. 8, the relationship from n-alkanes and melting point is

$$
\mathrm{m} . \mathrm{p} .=415 \frac{M}{M+95}-273
$$

where m.p. is the melting point and $M$ is the molecular weight. Therefore, by changing the composition of the paraffin wax it is possible to obtain different melting points. In general, the chemical characteristics of paraffin depend on its composition. The most interesting behavior of the paraffin can be detected when the temperature approaches the melting point: the wax starts to

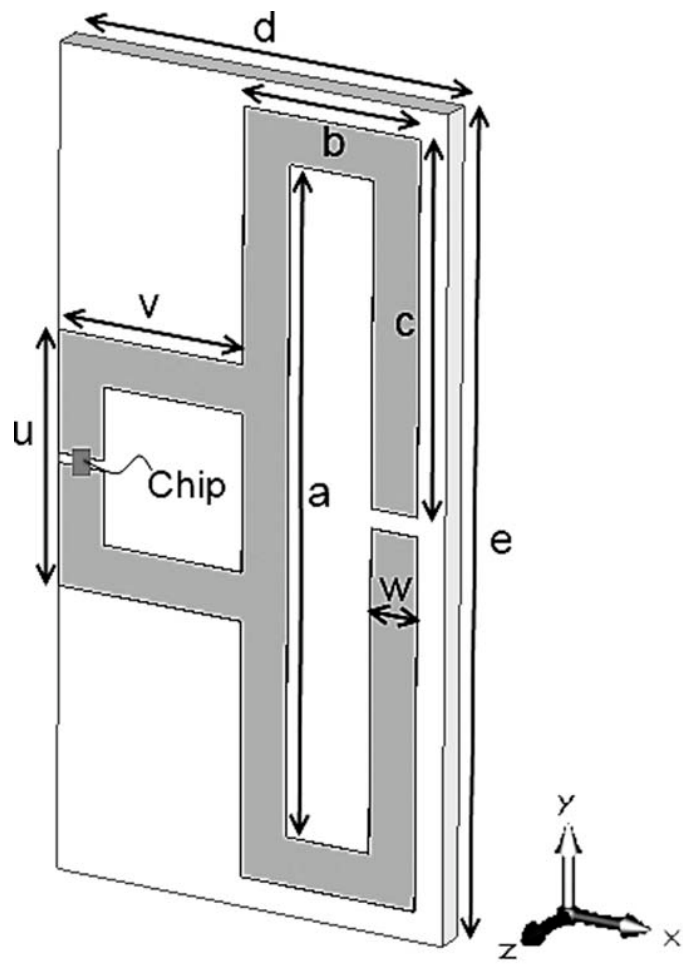

Figure 1 Antenna geometry and parameters 
TABLE 1 Values of the Geometrical Parameters in Millimeters

\begin{tabular}{cc}
\hline Parameter & Value $[\mathrm{mm}]$ \\
\hline$a$ & 68 \\
$b$ & 19 \\
$c$ & 38 \\
$d$ & 42 \\
$e$ & 84 \\
$u$ & 26 \\
$v$ & 20 \\
$w$ & 5 \\
\hline
\end{tabular}

change its molecular structure, density, volume, and dielectric properties [7]. Changes of this kind can alter the antenna's performance if the paraffin is used as its substrate. Paraffin, in this case, can be considered a passive temperature sensor for RFID tags. It is worth noticing that in this article the possibility to perform the analysis of the paraffin as a passive temperature sensor is not an aim of this work but it is the future of this research.

This article presents the performance of a highly purified paraffin wax: Paraplast [9], which has a melting point of $56^{\circ} \mathrm{C}$ and it is composed of $99 \%$ of pure paraffin. Relative permittivity and conductivity of Paraplast were measured over the frequency range from $800 \mathrm{MHz}$ to $1 \mathrm{GHz}$ with Agilent VNA E8358A, using the Agilent $85070 \mathrm{E}$ dielectric probe. To validate the dielectric probe measurements, the electromagnetic features of our paraffin sample have been experimentally evaluated with a specific parameter identification technique using numerical models and measurements on planar antennas, as explained in Ref. 10.

After the experimentation, the permittivity and dissipation factor of the paraffin have been assumed constant in the UHF RFID band from 860 to $960 \mathrm{MHz}: \varepsilon=2.1$ and $\tan \delta=9.8 \times$ $10^{-4}$ showing a good agreement with the values found in literature $([7,11,12])$ and low losses.
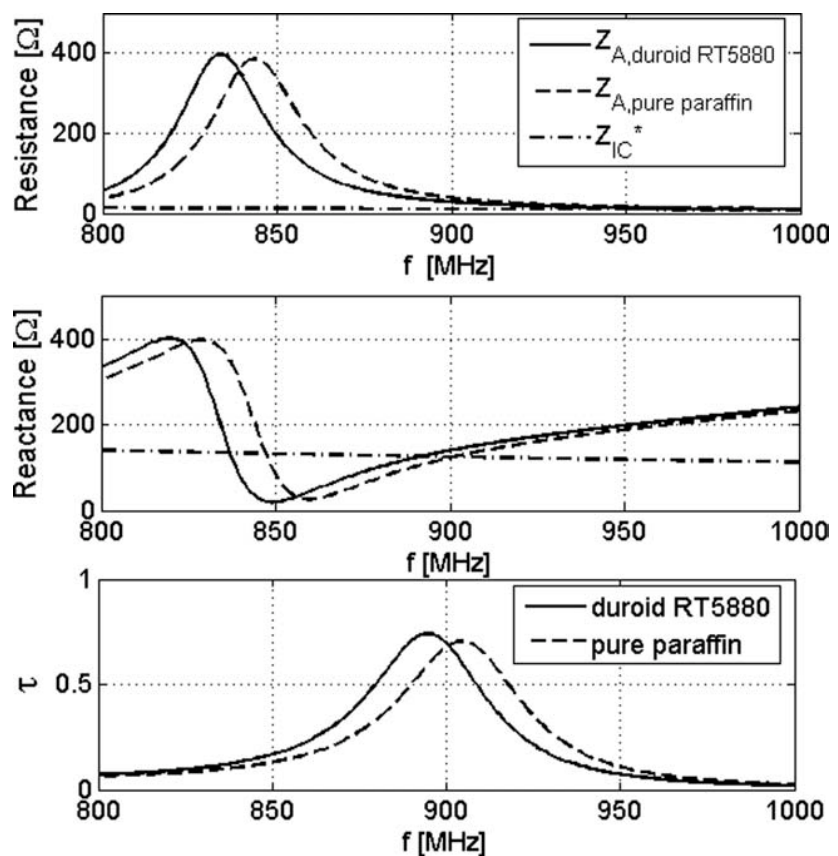

Figure 2 Simulated input impedance and power transmission coefficient of the paraffin and Duroid RT5880 tag with dimensions summarized in Table 1

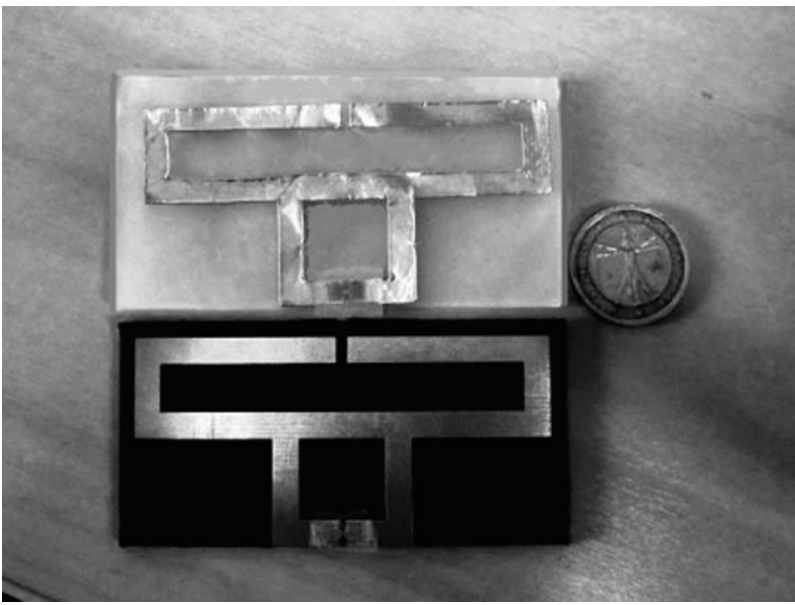

Figure 3 Prototypes of the pure paraffin tag (above) and Duroid RT5880 tag (below)

\section{PROPOSED TAG ANTENNA}

To validate and test the performance of paraffin as tag antenna substrate, a simple folded dipole tag was designed on two different substrates: a 3-mm thick layer of Paraplast and a 3-mm thick layer of Rogers/Duroid RT5880 [13], whose dielectric properties are close to those of paraffin: $\varepsilon=2.2$ and $\tan \delta=9 \cdot \times 10^{-4}$ [13].

The tag was designed and simulated using CST Microwave studio EM simulator. The Alien $\mathrm{H} 2$ IC with sensitivity $P_{\mathrm{IC}, 0}=$ $-14 \mathrm{dBm}$ was modeled by using a frequency dependent impedance model with impedance values found in the IC's datasheet [14]. In the model a parasitic capacitance of $0.2 \mathrm{pF}$ was also considered in parallel with the nominal IC impedance to take into account the presence of the IC strap impedance [15]. As this is the first prototype, the tags are not designed for any standard RFID frequency band. The tags are matched to a center frequency which allows good power transfer between the antenna and IC, around $900 \mathrm{MHz}$. This frequency is within the global passive RFID frequency band from 860 to $960 \mathrm{MHz}$.

The geometry of the chosen tag is a folded dipole on a substrate of 3-mm thick Duroid RT5880 and Paraplast, as shown in Figure 1 where the parameters of the antenna are reported. The IC is positioned in a $1 \mathrm{~mm}$ long gap in the middle of the Tmatch section.

The antenna's input impedance was changed with the Tmatching approach through the parameters $u$ and $v$ to achieve the conjugate matching with the IC impedance [16]. The electromagnetic tag performances have been optimized by maximizing the power transmission coefficient

$$
\tau=\frac{4 \mathrm{R}_{\mathrm{IC}} \cdot R_{\mathrm{A}}}{\left|\mathrm{Z}_{\mathrm{IC}}+Z_{\mathrm{A}}\right|^{2}}
$$

between the tag antenna and tag chip impedances, $Z_{\mathrm{A}}$ and $Z_{\mathrm{IC}}$, respectively. The numerical values of the tag's chosen dimensions are listed in Table 1 and simulation results of both paraffin and Duroid RT5880 tags are shown in Figure 2.

Both tags with pure paraffin and Duroid RT5880 substrate show a good matching results around $900 \mathrm{MHz}$. The power transmission coefficient is just slightly shifted due to the effect of different permittivity of the two substrates.

The first tag has been fabricated on Rogers/Duroid RT5880 by using the milling machine, whereas the second one has been 


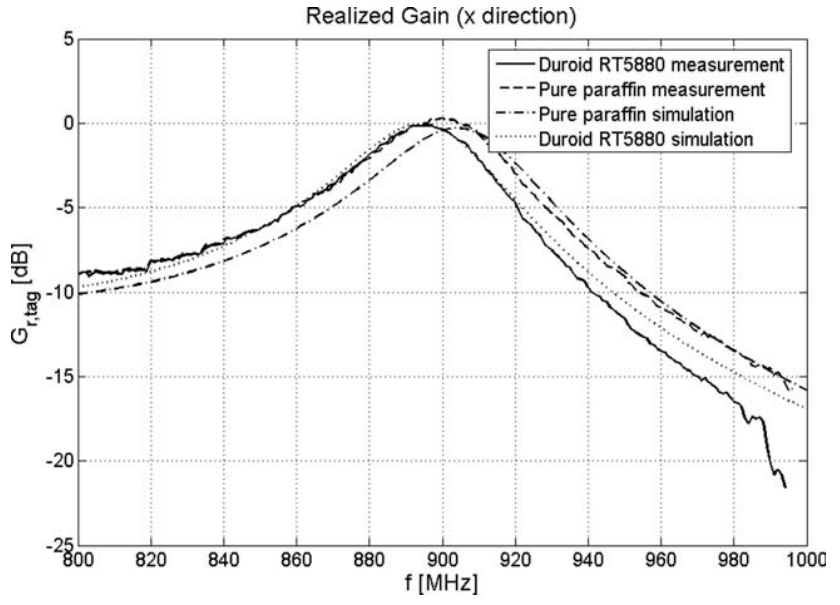

Figure 4 Simulated and measured realized tag antennas gain in the direction of the positive $x$-axis in Figure 1

made by manually placing a copper sheet on top of the pure paraffin layer. Both the prototype tags are shown in Figure 3.

\section{EXPERIMENTAL PERFORMANCES CHARACTERIZATION}

The electromagnetic performance of the tags have been experimentally characterized by measuring the realized gain $G_{\mathrm{r}, \mathrm{tag}}=$ $G_{\text {tag }} \cdot \tau$, where $G_{\text {tag }}$ is the radiation gain of the tag's antenna and $\tau$ is the power transmission coefficient.

For this purpose, Tagformance RFID measurement device [17], a measurement unit for RFID tag performance characterization, has been used to test the tag antennas in a compact anechoic chamber. The reader performs power-on tag sweeps between $800 \mathrm{MHz}$ and $1 \mathrm{GHz}$. A linearly polarized reader antenna sends the EPC Generation 2 query command to the tag under test, which then replies with the ID code. The realized gain of the tag can be derived from the measured power-on of the $\operatorname{tag} P_{\text {on }}$, which is the measured threshold power to activate the tag in the forward direction, regardless from the reader-tag distance.

The realized gain of the tag can be calculated from

$$
G_{\mathrm{r}, \mathrm{tag}}=\frac{P_{\mathrm{IC}, 0}}{L_{\mathrm{fwd}} P_{\mathrm{on}}}
$$

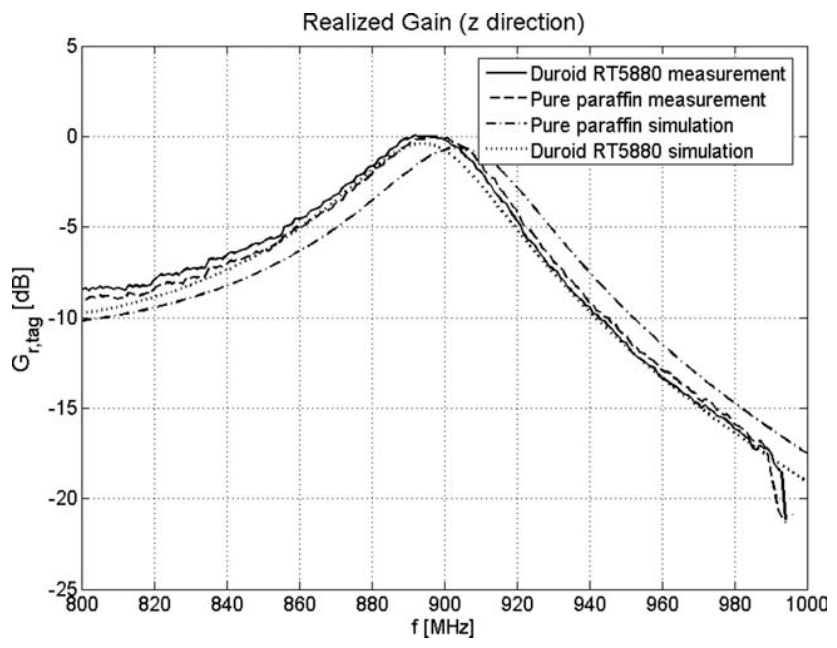

Figure 5 Simulated and measured realized tag antennas gain in the direction of the positive $z$-axis in Figure 1

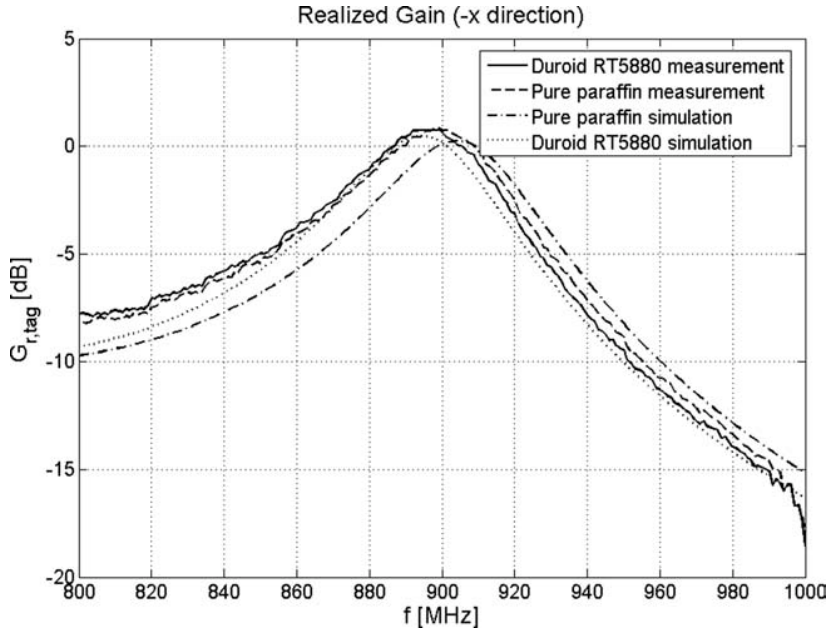

Figure 6 Simulated and measured realized tag antennas gain in the direction of the negative $x$-axis in Figure 1

where $L_{\text {fwd }}$ is the pathloss from the generator's output port to the input port of a hypothetic isotropic antenna placed at the tag's location, measured by using a calibration tag provided by the manufacturer, and $P_{\mathrm{IC}, 0}$ is the sensitivity of the IC, in our case equal to $-14 \mathrm{dBm}$.

Figures 4, 5, and 6 show the simulated and measured real- F4 ized gain versus frequency of the pure paraffin and Duroid RT5880 tags for three different directions. A good agreement can be noticed between measurements and simulations. Both of the tags with different substrates demonstrate the same performances and behavior. The measured normalized gain radiation patterns of paraffin and Duroid RT5880 tags, at the central operating frequency of $895 \mathrm{MHz}$, are presented in Figures 7 and 8 .

Figure 7 shows the $E$-plane ( $y-z$ plane) radiation pattern of the paraffin and Duroid RT5880 tags, whereas Figure 8 shows the $H$-plane ( $x-z$ plane) radiation pattern of the paraffin and Duroid RT5880 tags. As expected, both the antennas present a typical dipole radiation pattern. In addition, in this case, the

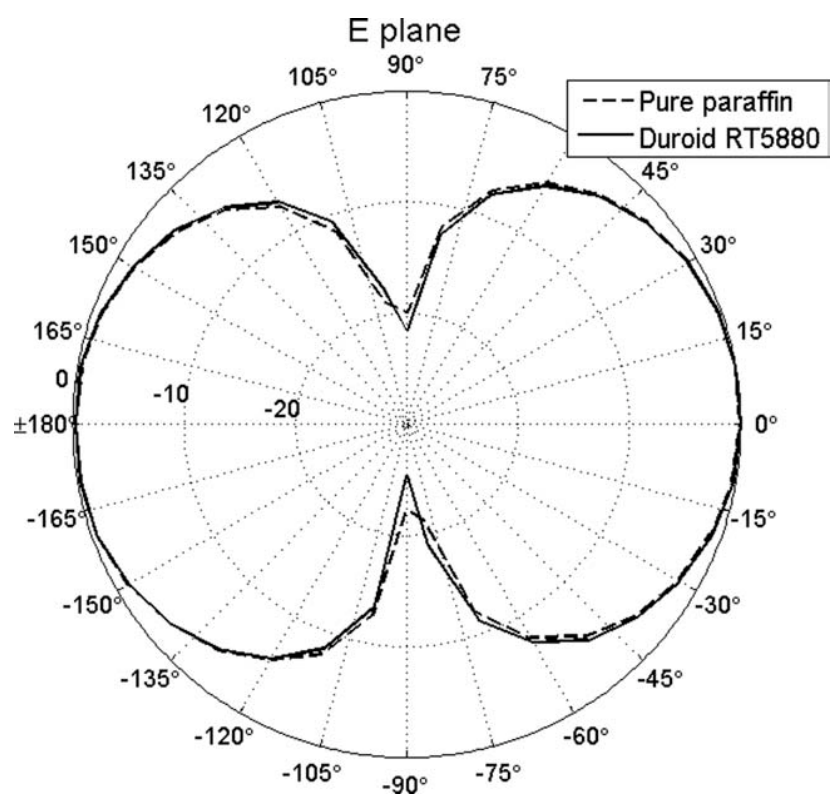

Figure 7 Measured $E$-plane ( $y-z$ plane of Fig. 1) pattern for the pure paraffin and Duroid RT5880 tags at the frequency of $895 \mathrm{MHz}$ 


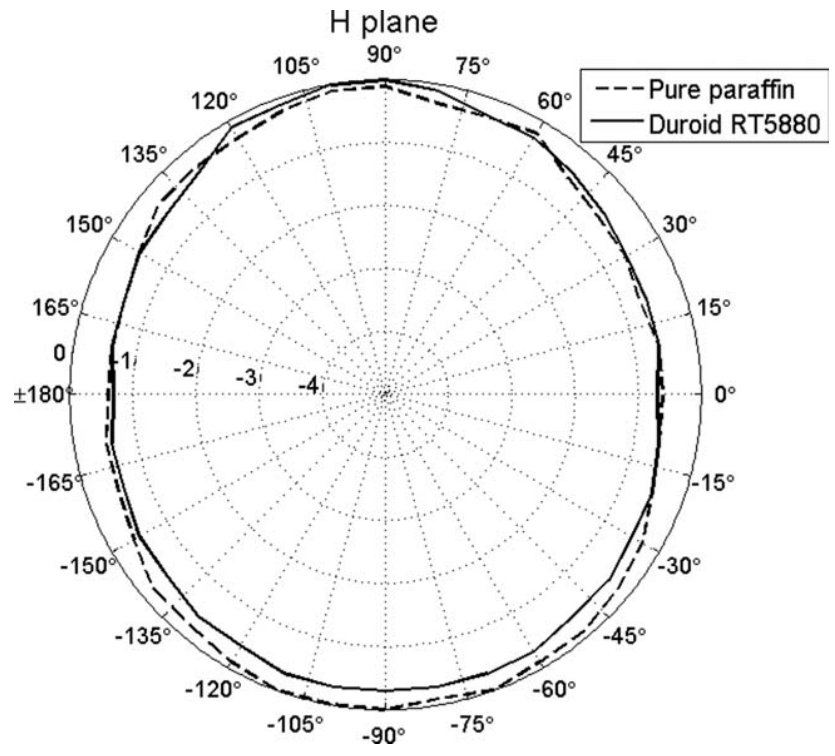

Figure 8 Measured $H$-plane $(x-z$ plane of Fig. 1) pattern for the pure paraffin and duroid RT5880 tags at the frequency of $895 \mathrm{MHz}$

behavior of the two tags, and thereby the characteristics of the two substrates under study, are very close.

\section{HEATING BEHAVIOR}

Since the aim of this research is to show how paraffin can be useful in temperature sensoristic applications, an inceptive experiment was performed to demonstrate the effects of the temperature change on the behavior of the paraffin as substrate. A dipole tag on a 0.165 -mm thick layer of FR4 was made with the same dimensions and shape reported in Figure 1 and Table 1. The tag was put on top of a 1-mm thick pure paraffin layer inside a vacuumed plastic bag, as shown in Figure 9. The plastic bag was thick $0.1 \mathrm{~mm}$ and its permittivity has been assumed equal to 3 .

The tag was measured with Tagformance RFID measurement device in a compact anechoic chamber at the room temperature. Subsequently, the tag was heated with a hot air blower until all the paraffin layer inside the vacuum bag was melted. Then after the solidification, it was measured again with Tagformance.

Figure 10 shows the results of these last measurements for one direction, before and after the heating. Measurements and

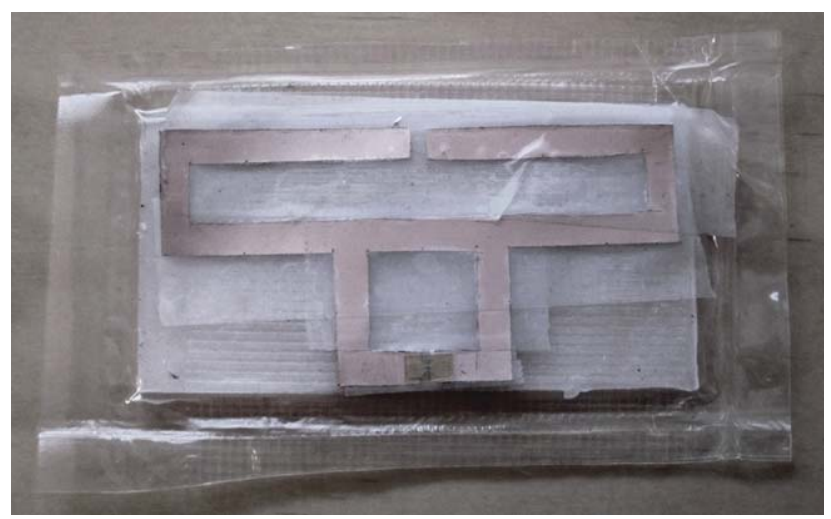

Figure 9 Dipole tag for heating measurements. [Color figure can be viewed in the online issue, which is available at wileyonlinelibrary.com]

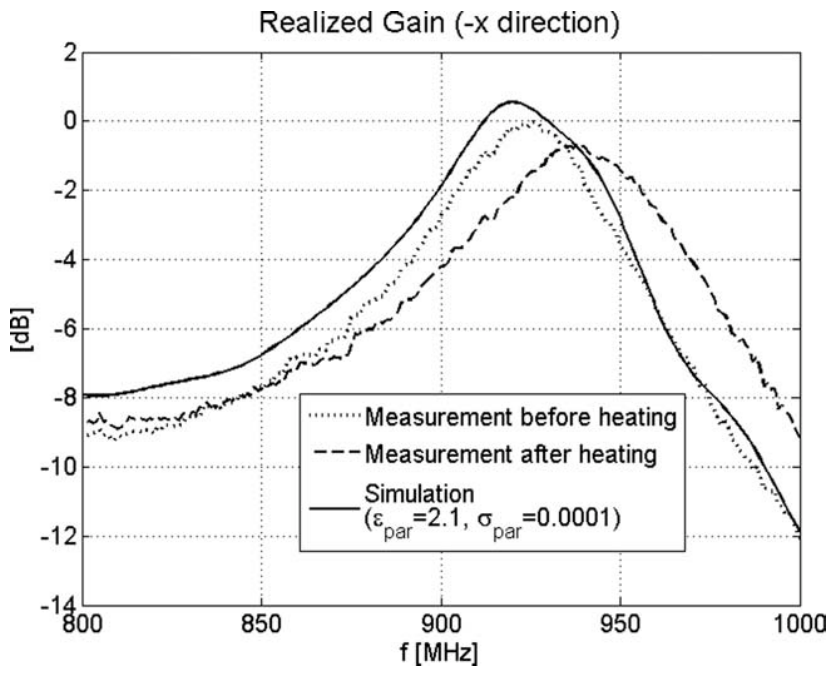

Figure 10 Simulated and measured realized tag antennas gain before and after the heating in the direction of the negative $x$-axis in Figure 1

simulations are in good agreement. The change in the properties of the paraffin can be noticed after the heating, which has an effect on the frequency and on the peak value of the realized gain.

The shift in frequency is about $15 \mathrm{MHz}$ while the peak value of the realized gain is reduced to $1 \mathrm{~dB}$ after the heating. These changes can be more easily detected in a sensor tag with a narrower band in order to measure also slight changes in the temperature.

\section{CONCLUSION AND FUTURE WORK}

In this article, we have presented a study of the performance of pure paraffin considered as tag antenna's substrate for UHF RFID systems. The permittivity of paraffin is close to Rogers/ Duroid 5800. The performances of two folded dipole tags in terms of realized gain have been simulated, measured, and compared. Results confirm that paraffin can be a suitable substrate for RFID sensors tags, low cost and with very low losses, comparable to the Duroid RT5880.

The dielectric properties, density and volume of paraffin change with temperature in the proximity of its melting point. A first experiment was performed to demonstrate the change of the paraffin's dielectric properties before and after the melting.

The future work will include investigation and test of a narrower band temperature sensor tag. In this case, paraffin can be considered both as antenna's substrate and passive temperature sensor for UHF RFID systems.

\section{ACKNOWLEDGMENTS}

This research work has been funded by the Finnish Funding Agency for Technology and Innovation (TEKES), Academy of Finland, Centennial Foundation for Finnish Technology Industries, Finnish Cultural Foundation, Nokia Foundation and High Technology Foundation of Satakunta, Finland. The author would like to thank T. Bjorninen for his support and help in this work.

\section{REFERENCES}

1. K. Finkenzeller, RFID handbook: Fundamentals and applications in contactless smart cards, radio frequency identification and nearfield communication, 2nd ed., Wiley, Hoboken, NJ, 2003. 
2. S. Caizzone, C. Occhiuzzi, and G. Marrocco, RFID radio-sensor integrating shape-memory alloys, IEEE Antennas and Propagation Society Symposium, Toronto, Canada, 2010.

3. C. Occhiuzzi and G. Marrocco, Pervasive body sensing: Implanted RFID tags for vascular monitoring, IEEE Antennas and Propagation Society Symposium, Toronto, Canada, 2010.

4. C. Occhiuzzi, S. Cippitelli, G. Marrocco, Modeling, design and experimentation of wearable RFID sensor tag, IEEE Trans Antennas Propag 58 (2010), 2490-2498.

5. C. Occhiuzzi and G. Marrocco, The RFID technology for neuroscience: Feasibility of limbs' monitoring in sleep diseases, IEEE Trans Info Tech Biomed 14 (2010), 37-43.

6. J. Virtanen, L. Ukkonen, T. Bjorninen, and L. Sydanheimo, Printed humidity sensor for UHF RFID systems, IEEE Sensors Applications Symposium (SAS), 2010, pp. 269-272.

7. M. Freund, R. Csikos, S. Keszthelyi, and G. Mozes, Paraffin products: Properties, technologies, applications, Dev Petrol Sci 14 (1983), 337.

8. A.H. Etessam and M.F.J. Sawyer, Znst Petrol 25 (1939), 253.

9. Paraplast datasheet [online]. Available at: http://www.2spi.com/ catalog/msds/msds02847.html

10. S. Sankaralingam and B. Gupta, Determination of dielectric constant of fabric materials and their use as substrates for design and development of antennas for wearable applications, IEEE Trans Instrum Meas 59 (2010), 3122-3130.

11. H. Fröhlich, Dielectric loss in paraffin-wax solutions, Proceedings of the Physical Society, Vol. 54, Sept. 1942.

12. S.G. Agaev and L.V. Taranova, Dielectric and electrophoretic properties of wax dispersions in the presence of pour depressants, Chem Technol Fuels and Oils 22, 541-545.

13. Rogers RT/duroid 5880 high frequency laminates datasheet [online]. Available at: http://www.rogerscorp.com/documents/606/ ac m/RT-duroid-5870-5880-Data-Sheet.aspx.

14. Alien Technology. Higgs 2 product overview [online]. Available at: http://www.alientechnology.com/docs/products/DS_H2. pdf.

15. T. Bjorninen, L. Ukkonen, and L. Sydanheimo, Design and noninvasive design verification of slot-type passive UHF RFID tag, in Proceedings of Radio and Wireless Symposium (RWS), New Orleans, LA, 2010, pp. 135-135.

16. G. Marrocco, The art of UHF RFID antenna design: impedancematching and size-reduction techniques, IEEE Antennas Propag Mag 50 (2008).

17. Voyantic, RFID measurements solutions, available at: http:// www.voyantic.com.

(C) 2011 Wiley Periodicals, Inc.

\section{TRANSMISSIVE FIBER OPTICAL DISPLACEMENT SENSOR USING CUTTING PLATE}

Xiaogen Hu, Yuhe Li, and Yanxiang Chen

State Key Laboratory of Precision Measurement Technology and

Instruments, Department of Precision Instruments and

Mechanology, Tsinghua University, Beijing 100084, China;

Corresponding author: hxg05@mails.tsinghua.edu.cn

\section{Received 5 May 2011}

ABSTRACT: Intensity modulated transmissive fiber optical displacement sensor is introduced and its performance is investigated. In the experiments, the relative error is $4 \%$ and the linearity is higher than $99.2 \%$ with source light intensity stable. The relative error is $0.2 \%$ with the source light intensity changed at a certainly frequency. (C) 2011 Wiley Periodicals, Inc. Microwave Opt Technol Lett 54:446-448, 2012; View this article online at wileyonlinelibrary.com. DOI 10.1002/ mop. 26586
Key words: fiber; displacement sensor; transmissive; intensity modulated; cutting plate

\section{INTRODUCTION}

Fiber has been greatly developed in the past three decades, and now is widely used in communication and sensing area [1]. When applied in sensing area, fiber shows its good characteristics, such as small size, lightweight, immunity to electromagnetic interference, passive (all dielectric) composition, high temperature performance, large bandwidth, high sensitivity, environmental ruggedness, and the ability of distributed sensing. What's more, fiber seems to be a kind of almighty sensor and could be applied in measuring lots of physical quantities, such as acceleration, electric and magnetic, temperature, pressure, acoustics, vibration, linear and angular position, strain, humidity, viscosity, chemical, etc [2, 3].

Fiber optical displacement sensor (FODS) is one of those fiber optic sensors. As the technology develops, the industry and research work call for high precision, large measure scale, lightweighting and disturbing-free displacement sensor. FODS performs well and is likely to be a suitable one to satisfy all the needs above. Intensity modulated FODS is one special kind of FODS. It is based on the modulation of light intensity between two fibers. Intensity modulated FODS is proved to be a type of high sensitivity displacement sensor from a theoretical prospective $[4,5]$. The sensing characteristic is determined by the geometrical parameters including fiber aperture, radius of fiber core, lateral separation of the transmitting and receiving fibers, the angle between the two fibers, and the reflector curvature radius [6]. More accurate mathematical models are also presented to describe such alternative sensing structures and analyze their sensing characteristics [7-9].

In this paper, transmissive intensity modulated FODS using cutting plate is introduced. A modulated light supplying method is presented to enhance the accuracy. In these experiments, laser diode is applied to be the light source, while the fibers are plastic ones. The light intensity is periodically changed at a certain frequency. A cutting plate is placed between two plastic fibers to modulate the intensity of light. The modulated light can effectively eliminate the noise induced by ambient light. The experimental results proved it to be accurate. What's more, a mathematical model depicting light intensity distribution is derived.

\section{EXPERIMENTAL SETUP}

A schematic diagram for lateral displacement measurement, which is of changeable measure scale and changeable measurement precision, is shown in Figure 1. The laser power is supplyed by a DAC module. Light is transmitted from laser, then coupled into transmitting fiber by lens. The light comes out of the back tip of transmitting fiber, through the lens, then becomes parrale light. Between the lens on transmitting fiber

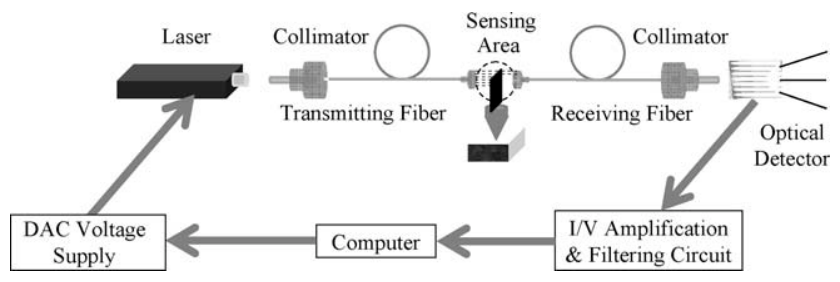

Figure 1 Schematic diagram of transmissive fiber optical displacement sensor 